\section{Ganzkörperwärmebehandlung - Warme Bäder gegen Depression?}

\section{Vielleicht sollten sich Depressive öfter mal ein heißes Bad gönnen: Eine Ganz- körperwärmebehandlung scheint jedenfalls die Stimmung deutlich zu heben - sogar über mehrere Wochen hinweg.}

$M_{2}^{\circ}$ oderne Antidepressiva können zwar vielen Patienten helfen, allerdings dauert es in der Regel zwei oder mehr Wochen, bis sich die stimmungsaufhellende Wirkung bemerkbar macht. Forscher suchen daher nach schneller wirksamen Verfahren, und dabei gehen sie mitunter sehr ungewöhnliche Wege. So konnten US-Wissenschaftler um Dr. Clemens Janssen von der Universität in Madison in einer kontrollierten Studie erstmals zeigen, dass eine Ganzkörperwärmebehandlung die Stimmung von Depressiven nachhaltig aufhellt.

Die Forscher begründen ihren Therapieversuch mit Beobachtungen, wonach eine als angenehm empfundene Wärme bestimmte Hirnregionen wie den mittleren orbitofrontalen Kortex, das vordere Cingulum oder das ventrale Striatum aktiviert - Bereiche, in denen Depressive häufig eine verminderte Aktivität aufweisen. Ließe sich diese durch ein wohdie Depression zurückgehen. Eine erste Studie mit 16 Depressiven wies in diese trollgruppe. Da die Placeboeffekte bei Depressionstherapien bekanntermaßen sehr ausgeprägt sind, wiederholten die liges Wärmegefühl verstärken, könnte Richtung, allerdings gab es keine Kon-
Forscher den Versuch mit einer Gruppe, die eine Scheinbehandlung erhielt.

\section{Körper auf $38,5^{\circ} \mathrm{C}$ erhitzt}

Die Wissenschaftler konnten 30 Patienten mit einer moderaten Depression gewinnen. Der Hamilton-Depressionsscore (HAMD17) lag zu Beginn bei rund 22 Punkten, die aktuelle Depressionsepisode hielt im Schnitt seit zwei Jahren an. Alle Depressiven durften sich einer Behandlung mit einem Ganzkörperhyperthermie-Gerät unterziehen. Sie machten es sich dabei auf einer Art Liege bequem, während Heizspiralen und Infrarotlampen über ihnen den Körper erhitzen. In der Gruppe mit Scheintherapie blieben die Infrarotstrahler jedoch aus. Stattdessen wurden nur die Heizspiralen etwas aktiviert, um den Probanden das Gefühl einer Wärmebehandlung zu vermitteln. Tatsächlich waren fast drei Viertel der Depressiven in der Kontrollgruppe nach der Intervention der Auffassung, die Wärmetherapie und nicht die Scheinbehandlung bekommen zu haben. In der Gruppe mit aktiver Therapie glaubte jedoch nur ein Patient, er habe die Scheintherapie erhalten.

Insgesamt erhielten 16 der Patienten die aktive und 14 die Scheintherapie. In der Nachbeobachtungszeit von sechs Wochen sollten die Patienten auf Antidepressiva verzichten.

Ziel war eine Kernkörpertemperatur von 38,5 Grad, sie wurde in der Gruppe mit aktiver Therapie nach knapp zwei Stunden erreicht. Sobald die Fieberkurve auf diesen Wert gestiegen war, schalteten die Studienärzte sämtliche Lampen $\mathrm{ab}$ und ließen die Patienten noch eine Stunde zur Abkühlung liegen. Die Scheinbehandlung dauerte vergleichbar lange, auch danach mussten die Probanden noch eine Stunde liegen bleiben.

Im Schnitt stieg die Körpertemperatur in der Gruppe mit aktiver Behandlung um $1,9^{\circ} \mathrm{C}$ auf $38,9^{\circ} \mathrm{C}$, in der Kontrollgruppe um $0,8^{\circ} \mathrm{C}$ auf $37,7^{\circ} \mathrm{C}$. Schon nach einer Woche zeigten sich statistisch signifikante Unterschiede zwischen den beiden Gruppen: Mit aktiver Therapie war der HAMD17-Wert um 6,1 Punkte gesunken, mit der Scheinbehandlung nur um 1,9 Punkte. Nach zwei Wochen fanden die Forscher einen Rückgang um jeweils 8,0 und 4,0 Punkte, in den folgenden vier Wochen blieben die HAMD17-Scores in beiden Gruppen relativ stabil. Bei den Nebenwirkungen hauptsächlich Kopfschmerzen, Fatigue und Mundtrockenheit - gab es keine signifikanten Differenzen zwischen den beiden Gruppen.

Nach diesen Daten, so schreiben die Forscher um Janssen, scheint eine einmalige Ganzkörperwärmetherapie die Stimmung anhaltend aufzuhellen. Allerdings solle man die Resultate nicht überbewerten - die Effektstärken waren geringer als das üblicherweise in Studien mit Antidepressiva der Fall ist. Die Wärmebehandlung kann eine spezifische antidepressive Behandlung folglich nicht ersetzen. Ein Manko ist zudem die geringe Teilnehmerzahl. Unklar ist zudem, ob die Art und Weise der Hyperthermie einen Einfluss auf das Ergebnis hat. Falls nicht, müssten sich ähnliche Effekte auch in der Badewanne oder mit einem Sonnenbad erzielen lassen.

(TM)

Janssen CW et al. Whole-Body Hyperthermia for the Treatment of Major Depressive Disorder. A Randomized Clinical Trial. JAMA Psychiatry 2016; epub 12.5.16, 2016. doi:10.1001/jamapsychiatry.2016.1031 